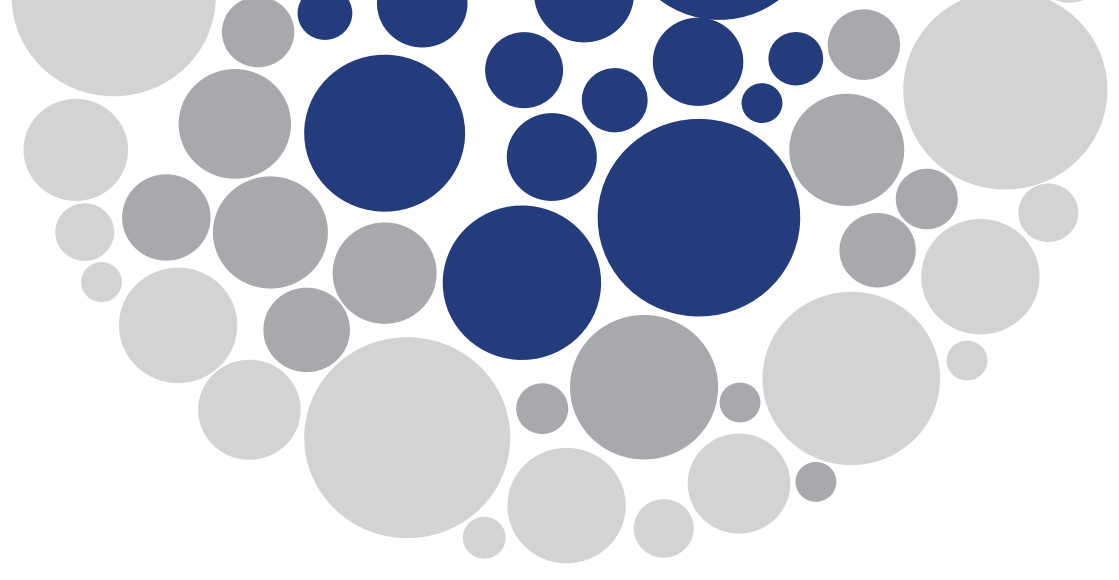

\title{
Análise das dissertações defendidas no Programa de Pós-Graduação em Geografia da UFF/Campos: contribuição para a autoavaliação do programa
}

\author{
Marcelo Werner da Silva ${ }^{a}$ \\ a Coordenador do Programa de Pós-Graduação em Geografia da UFF/CAMPOS. E-mail: marcelows@id.uff.br
}

\section{Introdução}

Este texto é uma contribuição para a avaliação do Programa de Pós-Graduação em Geografia (PPG) da Universidade Federal Fluminense, em Campos dos Goytacazes (UFF/Campos), pois, atualmente, os órgãos reguladores da pós-graduação exigem que se faça uma autoavaliação. Para tanto, vamos explicitar esses critérios autoavaliativos e discorrer sobre algumas características das dissertações desenvolvidas no PPG desde seu início, que se deu em 2014.

\section{A autoavaliação da pós-graduação brasileira}

Para a Coordenação de Aperfeiçoamento de Pessoal de Nível Superior (CAPES), a autoavaliação é 
formativo, de aprendizagem. Uma vez que é planejada, conduzida, implementada e analisada por pessoas elas próprias formuladoras e agentes das ações a serem avaliadas, a autoavaliação possibilita uma reflexão sobre contexto e políticas adotadas, além da sistematização dos dados que levam à tomada de decisão (COORDENAÇÃO DE APERFEIÇOAMENTO DE PESSOAL DE NÍVEL SUPERIOR, 2019, p. 7).

Portanto, trata-se de um processo preparado e gerido pela própria comunidade acadêmica com "a titularidade de avaliação" (COORDENAÇÃO DE APERFEIÇOAMENTO DE PESSOAL DE NÍVEL SUPERIOR, 2019, p. 7) que envolve no processo avaliativo "a participação de distintos atores da academia ou externos a ela (docentes, discentes, egressos, técnicos e outros), nos níveis hierárquicos diversos, dos estratégicos aos mais operacionais" (ibidem). Ainda segundo a CAPES, do trabalho participativo resultam melhores produtos no que se refere à autoavaliação.

O objetivo da CAPES com a autoavaliação é realizar o acompanhamento da forma como cada programa de pós-graduação está conduzindo sua autoavaliação: "Desta maneira, cada programa poderá propor um delineamento de autoavaliação apto a captar aspectos pertinentes a sua missão e seus objetivos, incluindo aqueles relativos à sua inserção no contexto social/internacional e a suas escolhas científicas específicas" (COORDENAÇÃO DE APERFEIÇOAMENTO DE PESSOAL DE NÍVEL SUPERIOR, 2019, p. 9).

No caso específico da área de Geografia, a que o Programa de Pós-Graduação em Geografia da UFF/CAMPOS (PPG) está vinculado, a ficha de avaliação, em seu item 1.4, consigna que serão avaliados "Os processos, procedimentos e resultados da autoavaliação do programa, com foco na formação discente e produção intelectual" (COORDENAÇÃO DE APERFEIÇOAMENTO DE PESSOAL DE NÍVEL SUPERIOR, 2020).

Deste modo, neste texto, vamos dar ênfase na produção intelectual do PPG, apresentando as dissertações produzidas. No entanto, para um melhor conhecimento do Programa, faremos, inicialmente, um pequeno histórico de sua criação.

\section{Histórico e Caracterização do Programa de Pós-Graduação em Geografia da UFF/Campos (PPG)}

O curso de mestrado em Geografia na UFF/Campos foi criado em 2013, apenas quatro anos após a criação do curso de graduação em Geografia, ocorrido em 2009, dentro do Programa de Apoio a Planos de Reestruturação e Expansão das Universidades Federais (REUNI).

O curso foi estruturado em uma área de concentração chamada "Análise Regional e Ambiental", a qual, por sua vez, é dividida em duas linhas de pesquisa: "Análise Regional, Dinâmicas Territoriais e Escalas" e "Sistemas Naturais, Geotecnologias e Demografia". Na proposta do curso, estão consignados os objetivos da área de concentração:

A definição de região parte do princípio da diferenciação de áreas. Por outro lado, o discurso da globalização prega a homogeneização dos espaços. É na interseção das questões relacionadas às especificidades espaciais e na globalização que as questões regionais tomaram novo fôlego nestas primeiras décadas do século XXI. As diversidades territoriais expressas, tanto pelas desigualdades econômicas, quanto pelas diferenças culturais, ampliaram os interesses dos geógrafos em explicar esses fenômenos que podem ser lidos a partir das relações entre fenômenos locais e globais que produzem, 
ao mesmo tempo territórios-zona onde prevalece a lógica política, territórios-rede conformados pela lógica econômica e os aglomerados de exclusão sob a égide da lógica social. Tratam-se, portanto dos espaços onde se reconhecem as articulações dos múltiplos agentes em diferentes escalas de ação. $\mathrm{Na}$ análise regional considera-se os aspectos ambientais em diferentes olhares: sejam nos estudos da evolução, dinâmica e processos dos diferentes componentes dos sistemas naturais a partir de diagnósticos e monitoramentos, ou numa perspectiva ampla, reconhecendo a análise ambiental como a integração de fenômenos sociais, políticos, econômicos, culturais e a natureza, em um recorte espacial específico. As relações inter-escalares dos sujeitos; das agências; das empresas e dos Estados-Nação, redefinem as regiões frente aos circuitos da globalização (UNIVERSIDADE FEDERAL FLUMINENSE, 2021).

\section{A linha de pesquisa de "Análise Regional, Dinâmicas Territoriais e Escalas",}

Fundamenta-se na execução de estudos regionais voltados a discussão ou elaboração de aspectos teóricos, metodológicos e epistemológicos em geografia. Essa linha de pesquisa propõe estudos sobre as dinâmicas territoriais, processos espaciais, instrumentos e políticas voltados à análise regional em suas diferentes escalas (do local ao global). Aborda as estruturas produtivas, os fluxos econômicos, as estratégias de gestão e de governança, os efeitos da globalização, da reestruturação produtiva, dos aspectos culturais, definidores de identidades regionais; além das interfaces entre as dinâmicas territoriais e as questões sociais e ambientais (UNIVERSIDADE FEDERAL FLUMINENSE, 2021).

Por sua vez, a linha de "Sistemas Naturais, Geotecnologias e Demografia" está ligada aos três eixos temáticos que estão identificados em seu nome, articulados da maneira exposta a seguir:

As geotecnologias envolvem a criação de cenários, modelos e análises espaço-temporais de uso e ocupação das terras aplicando diferentes metodologias na adoção das Geotecnologias nas diferentes categorias de análise da Geografia. A demografia aborda estudos sobre a distribuição da população humana no espaço assim como sua estrutura, envolvendo estudos sobre os efeitos territoriais de políticas populacionais; sobre problemas relacionados ao trinômio população, desenvolvimento e ambiente; e a elaboração de análises e diagnósticos demográficos em várias escalas, envolvendo projeções de população, estimativas de demandas sociais e construção de indicadores sociais. Os Sistemas Naturais abordam estudos voltados para o conhecimento e análise ambiental, envolvendo a evolução, dinâmica e os processos dos diferentes sistemas naturais ao longo das quatro escalas temporais de análise: geológica, histórica, presente e futura, que dão suporte a diagnósticos e monitoramentos, assim como subsidiam o processo de uso e ocupação das terras mitigando os impactos ambientais. A compreensão dos sistemas naturais subsidiam políticas e planos de zoneamento ambiental de Unidades de Conservação da Natureza, bem como dão suporte a estudos que se preocupam com os efeitos advindos dos conflitos e impactos ambientais nos diferentes ambientes costeiros e continentais (UNIVERSIDADE FEDERAL FLUMINENSE, 2021).

Portanto, unindo a área de concentração com as duas linhas de pesquisa, podemos identificar os objetivos do PPG:

[...] aprimorar a formação de pesquisadores em nível de mestrado visando o desenvolvimento acadêmico e científico, assim como a formação de profissionais de alto nível para o exercício das atividades de ensino, pesquisa e extensão em geografia em todo território nacional. Além disso, espera-se que o egresso do Programa acumule 
capital humano geral e específico para construir, aplicar e divulgar conhecimento, contribuindo para o desenvolvimento da ciência geográfica no Brasil. Esse profissional deverá ainda estar apto a apreender os conteúdos teórico-metodológicos e aplicá-los aos problemas da realidade no seu exercício profissional, sejam eles oriundos de demandas regionais ou nacionais (UNIVERSIDADE FEDERAL FLUMINENSE, 2021).

Levando em consideração a maneira como o curso foi estruturado, vamos analisar as dissertações defendidas no PPG desde sua criação até o final do ano de 2020, de forma a constatar sua adequação à área de concentração e às linhas de pesquisa.

\section{As dissertações defendidas no PPG no período 2016-2020}

No Anexo 1, estão relacionadas todas as 66 dissertações defendidas desde a criação do PPG. Como sua primeira turma foi selecionada em 2014, as primeiras dissertações foram defendidas só no ano de 2016.

Na Tabela 1, estão as dissertações separadas por ano e por linha de pesquisa. Podemos perceber uma predominância da linha de "Análise Regional, Dinâmicas Territoriais e Escalas". Porém, se analisarmos apenas os anos de 2016, 2017 e 2018, chegamos a números bastante próximos: 53,5\% para a linha de "Análise Regional, Dinâmicas Territoriais e Escalas" e 46,5\% para a linha de "Sistemas Naturais, Geotecnologias e Demografia". Contudo, incluindo os anos de 2019 e 2020 , os números passam a $62 \%$ e $38 \%$, respectivamente. Somando todo o período, temos uma média de 13 dissertações defendidas por ano.

Em relação ao número de docentes, o PPG possuía, em 2014, 16 docentes, dos quais 14 permanentes, sendo 6 pertencentes à linha de "Análise Regional, Dinâmicas Territoriais e Escalas" e 8 pertencentes à linha de "Sistemas Naturais, Geotecnologias e Demografia", e 2 colaboradores, sendo um em cada uma das linhas de pesquisa.

Em 2020, o PPG contou 15 docentes permanentes, dos quais 8 pertenciam à linha de "Análise Regional, Dinâmicas Territoriais e Escalas" e 7 estavam ligados à linha de "Sistemas Naturais, Geotecnologias e Demografia", e 2 colaboradores, cada um pertencente a uma das linhas.

Portanto, de 2014 para 2020, houve um aumento na quantidade de docentes da primeira linha de pesquisa e uma diminuição da segunda, o que talvez ajude a explicar o menor número de defesas em "Sistemas Naturais, Geotecnologias e Demografia".

Tabela 1 - Número de dissertações defendidas no PPG no período 2016-2020, separados por linha de pesquisa.

\begin{tabular}{|llll}
\hline & $\begin{array}{l}\text { Análise Regional, } \\
\text { Dinâmicas Territoriais e } \\
\text { Escalas }\end{array}$ & $\begin{array}{l}\text { Sistemas Naturais, } \\
\text { Geotecnologias e } \\
\text { Demografia }\end{array}$ & TOTAL \\
\hline 2016 & 3 & 4 & 7 \\
\hline 2017 & 11 & 9 & 20 \\
\hline 2018 & 9 & 7 & 16 \\
\hline 2019 & 6 & 3 & 9 \\
\hline 2020 & 12 & 2 & 14 \\
\hline Subtotal por linha & 41 & 25 & 66 \\
\hline
\end{tabular}

Fonte: elaborada pelo autor. 


\section{Recortes Espaciais}

Muito importante em Geografia é delimitar os recortes espaciais da pesquisa, os marcos espaciais em que a pesquisa ocorrerá. Castro $(2014$, p. 88$)$ entende a escala geográfica "como um problema operacional fundamental na definição do recorte espacial significativo para a análise do fenômeno na pesquisa em geografia”. Para ela, refletir sobre

[...] a escala na geografia é necessariamente tomar o fenômeno e sua extensão espacial, independente do ator responsável por ele, considerando que este é o problema central para qualquer pesquisa na disciplina. $O$ recurso de pensar a escala permite analisar o fenômeno a partir da medida da sua significância, isto é, da extensão que lhe dá sentido. Deixando claro que para a pesquisa nem o fenômeno, nem a escala de análise são dados da natureza, mas escolhas intelectuais fortemente influenciadas pelas matrizes teóricas dos pesquisadores e pelos seus contextos sociais.

Portanto, a análise que aqui realizamos busca averiguar as escolhas realizadas pelos agora egressos do PPG na construção de seus objetos teóricos e empíricos de investigação, podendo, então, verificar se os objetivos iniciais do PPG, de voltar-se para as questões da região de Campos dos Goytacazes e das regiões vizinhas do Norte e Noroeste Fluminenses, foram, de fato, priorizados nos recortes espaciais das pesquisas desenvolvidas.

$\mathrm{Na}$ Tabela 2, temos os recortes espaciais identificados nas dissertações defendidas no PPG. Para tanto, consideramos a indicação espacial presente no título. Já nos casos dos trabalhos em que tal indicação não estava definida, o recorte espacial foi apontado como "indefinido".

Simplificando os dados da Tabela 2, chegamos à Tabela 3, fazendo a ressalva de que existe a sobreposição de alguns trabalhos, sendo que, nesses casos, foi privilegiada a escala mais próxima a Campos dos Goytacazes/RJ.

Encontramos, portanto, 24 indicações da cidade de Campos dos Goytacazes/RJ, local de funcionamento do PPG, correspondendo a 36,36\% das dissertações; Onze trabalhos não tiveram indicação do recorte espacial no título (16,67\%), sendo considerados como "indefinidos"; Três indicaram claramente a região Norte Fluminense como seu recorte espacial; Quatro apontaram cidades da região Norte Fluminense (São João da Barra, com três 3 indicações, e Carapebus, com uma1); e um que indicou o Rio Paraíba do Sul, no trecho referente às regiões Norte e Noroeste Fluminenses; dois trabalhos indicaram a região Noroeste Fluminense (um sobre a cidade de Itaperuna; e o outro envolveu uma

Tabela 2 - Recortes espaciais identificados nas dissertações defendidas no PPG, 2016-2020.

\begin{tabular}{|llll}
\hline 1 & Indefinido & 34 & Campos dos Goytacazes \\
\hline 2 & Rio de Janeiro (estado) & 35 & Indefinido \\
\hline 3 & Porto do Açu & 36 & Campos dos Goytacazes \\
\hline 4 & Itaperuna/RJ & 37 & Indefinido \\
\hline 5 & Indefinido & 38 & Campos dos Goytacazes \\
\hline 6 & Indefinido & 39 & São João da Barra/RJ \\
\hline 7 & Indefinido & 40 & Indefinido \\
\hline
\end{tabular}

Fonte: elaborada pelo autor com dados do Anexo 1.

Obs.: A numeração se refere à ordem das dissertações apresentadas no Anexo 1. 
Tabela 2 - Continuação...

\begin{tabular}{|c|c|c|c|}
\hline 8 & Petrópolis/RJ & 41 & Araruama/RJ \\
\hline 9 & Campos dos Goytacazes e Macaé & 42 & Campos dos Goytacazes \\
\hline 10 & Guiné-Bissau (África) & 43 & Campos dos Goytacazes \\
\hline 11 & Cabo Frio/RJ (Rio de Janeiro) & 44 & Niterói/RJ \\
\hline 12 & Campos dos Goytacazes & 45 & Campos dos Goytacazes \\
\hline 13 & Espírito Santo & 46 & Espera Feliz/MG \\
\hline 14 & Espírito Santo & 47 & Brasil \\
\hline 15 & Campos dos Goytacazes & 48 & Porto Alegre/RS \\
\hline 16 & Campos dos Goytacazes & 49 & Campos dos Goytacazes \\
\hline 17 & Indefinido & 50 & Indefinido \\
\hline 18 & Rio de Janeiro (estado) & 51 & Norte Fluminense \\
\hline 19 & Brasil & 52 & Campos dos Goytacazes \\
\hline 20 & Suzano/SP & 53 & Viçosa/MG \\
\hline 21 & Rio de Janeiro (estado) & 54 & Rio de Janeiro (estado) \\
\hline 22 & Indefinido & 55 & Porto Seguro/BA \\
\hline 23 & Campos dos Goytacazes & 56 & Norte Fluminense \\
\hline 24 & Campos dos Goytacazes & 57 & Campos dos Goytacazes \\
\hline 25 & $\begin{array}{l}\text { Bom Jesus do Itabapoana/RJ e Bom Jesus } \\
\text { do Norte/ES }\end{array}$ & 58 & $\begin{array}{l}\text { Rio Paraíba do Sul (regiões Norte } \\
\text { Fluminense e Noroeste Fluminense) }\end{array}$ \\
\hline 26 & Indefinido & 59 & Uberaba/MG \\
\hline 27 & Campos dos Goytacazes & 60 & Carapebus/RJ \\
\hline 28 & Campos dos Goytacazes & 61 & São João da Barra/RJ \\
\hline 29 & Campos dos Goytacazes & 62 & Campos dos Goytacazes \\
\hline 30 & Campos dos Goytacazes & 63 & Norte Fluminense \\
\hline 31 & Campos dos Goytacazes & 64 & Campos dos Goytacazes \\
\hline 32 & Campos dos Goytacazes & 65 & Aparecida/SP \\
\hline 33 & Rio de Janeiro (estado) & 66 & Campos dos Goytacazes \\
\hline
\end{tabular}

Fonte: elaborada pelo autor com dados do Anexo 1.

Obs.: A numeração se refere à ordem das dissertações apresentadas no Anexo 1.

Tabela 3 - Classificação dos recortes espaciais encontrados na Tabela 2.

\begin{tabular}{lll}
\hline Recortes espaciais & Quantidade & Percentual \\
\hline Campos dos Goytacazes & 24 & $36,36 \%$ \\
\hline Região Norte Fluminense & 8 & $12,12 \%$ \\
\hline Região Noroeste Fluminense & 2 & $3,03 \%$ \\
\hline Rio de Janeiro (cidades e estado) & 9 & $13,64 \%$ \\
\hline Outros estados da região Sudeste & 7 & $10,61 \%$ \\
\hline Outras regiões brasileiras (Rio Grande do Sul e Bahia) & 2 & $3,03 \%$ \\
\hline Escala nacional & 2 & $3,03 \%$ \\
\hline Escala internacional (Guiné-Bissau) & 1 & $1,51 \%$ \\
\hline Recorte espacial indefinido & 11 & $16,67 \%$ \\
\hline TOTAL & 66 & $100,00 \%$
\end{tabular}

Fonte: elaborada pelo autor.

Observação: no caso de sobreposição de escalas, foi privilegiada a mais próxima da cidade de Campos dos Goytacazes/RJ. 


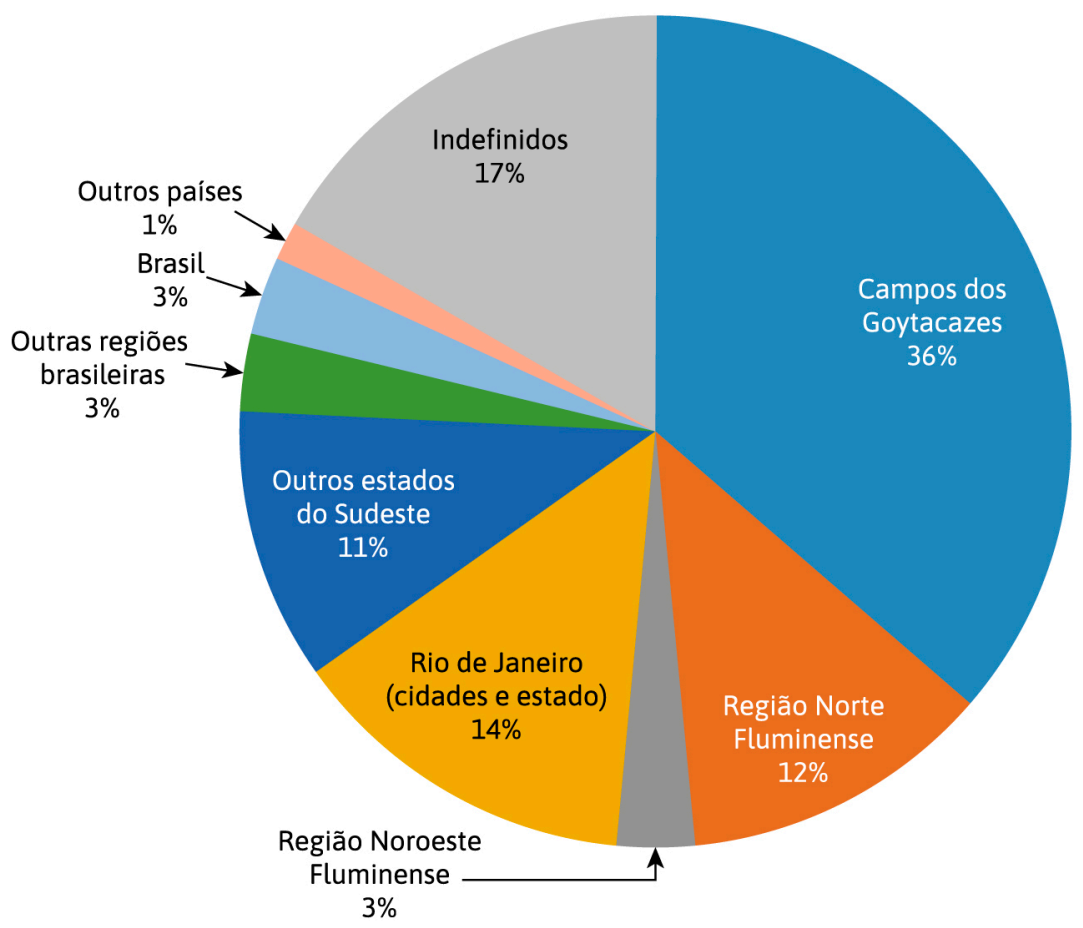

Figura 1 - Percentual dos recortes espaciais das dissertações do PPG, 2016-2020.

Fonte: elaborada pelo autor com dados da Tabela 3.

cidade do Noroeste Fluminense (Itaperuna) e outra uma cidade do estado do Espírito Santo). Portanto, somando os trabalhos da região Norte Fluminense com os da região Noroeste Fluminense, foram outros dez 10 trabalhos, que corresponderam a 15,15\% das dissertações. Além disso, nove trabalhos $(13,63 \%)$ realizaram como recorte espacial o estado do Rio de Janeiro ou cidades de outras regiões do estado, excluindo as regiões Norte e Noroeste Fluminense, que tiveram contabilização própria.

Sobre esses recortes espaciais mais próximos, destacamos aqui também: três trabalhos sobre o Espírito Santo (um já citado antes, que reuniu uma cidade capixaba com outra da região Noroeste Fluminense), três que envolveram cidades de Minas Gerais (Espera Feliz, Uberaba e Viçosa), dois referentes a cidades de São Paulo (Suzano e Aparecida), um sobre o Rio Grande do Sul (Porto Alegre) e um sobre a Bahia (Porto Seguro). Ainda, temos dois trabalhos da escala nacional e um da escala internacional, que versou sobre Guiné-Bissau (Figura 1).

\section{Considerações Finais}

Apresentamos, brevemente, alguns dados que podem servir para construir o histórico do curso, bem como contribuir para a melhoria de certos indicadores, analisando, por exemplo, a distribuição das dissertações defendidas em cada uma das linhas de pesquisa do curso. Deste modo, estamos colaborando com a autoavaliação exigidas pelos órgãos reguladores da pós-graduação brasileira.

A vocação regional do curso é comprovada quando vemos que $51,51 \%$ das dissertações apresentadas no PPG estão relacionadas à cidade de Campos dos Goytacazes ou às regiões 
Norte e Noroeste Fluminenses. Somando as referências ao estado do Rio de Janeiro e suas cidades, esse número sobre para $65,15 \%$ das dissertações.

Acrescentamos a isso a reflexão teórica, sempre necessária ao desenvolvimento da disciplina geográfica, presente nos trabalhos sem uma definição clara do recorte espacial. Destacamos ainda as menções aos estados do Espírito Santo e de Minas Gerais, que também podemos considerar como estando dentro da zona de abrangência do PPG.

Mesmo os recortes espaciais do Brasil e de estados como Rio Grande do Sul, São Paulo e Bahia mostram o espraiamento da reflexão teórica e prática do PPG, chegando até a escala internacional, com o trabalho sobre Guiné-Bissau, na África.

Portanto, com este trabalho, iniciamos uma reflexão sobre a produção do PPG, que pode ser estendida à realização de outras pesquisas e análises sobre as dissertações defendidas, servindo como indicadores para a melhoria constante do PPG em Geografia da UFF/Campos. Esse é o exigido na autoavaliação do PPG, tal qual é o objetivo de seus docentes, discentes e técnicos, sempre buscando uma educação gratuita e de qualidade, colaborando para a melhoria das condições de vida da população brasileira.

\section{Referências}

COORDENAÇÃO DE APERFEIÇOAMENTO DE PESSOAL DE NÍVEL SUPERIOR - CAPES. Autoavaliação de programas de Pós-graduação: grupo de trabalho. Brasília: CAPES, 2019. Disponível em: <https:// www.gov.br/capes/pt-br/centrais-de-conteudo/10062019-autoavaliacao-de-programas-de-pos-graduacaopdf>. Acesso em: 9 abr. 2021.

COORDENAÇÃO DE APERFEIÇOAMENTO DE PESSOAL DE NÍVEL SUPERIOR - CAPES. Ficha de avaliação. Brasília: CAPES, 2020. Disponível em: <https://www.gov.br/capes/pt-br/acesso-a-informacao/ acoes-e-programas/avaliacao/sobre-a-avaliacao/areas-avaliacao/sobre-as-areas-de-avaliacao/colegiode-humanidades/ciencias-humanas/geografia>. Acesso em: 9 abr. 2021.

CASTRO, I. E. Escala e pesquisa na geografia: problema ou solução? Revista Espaço Aberto: Revista do Programa de Pós-graduação em Geografia, Niterói, v. 4. n. 1, 2014.

UNIVERSIDADE FEDERAL FLUMINENSE - UFF. Programa de Pós-graduação em Geografia - PPG. Descrição do Programa. Niterói, 2021. Disponível em: <http://www.ppg.uff.br/index.php/area-deconcentracao/>. Acesso em 10 abr. 2021. 


\section{ANEXO 1 - DISSERTAÇÕES DEFENDIDAS NO PPG NO PERÍODO 2016-2020}

2020

ALVES, Hortencia de Jesus Rodrigues. Mirando as Tecnologias: aprendizagens de geografias vizinhas. Dissertação (Mestrado em Geografia) - Programa de Pós-Graduação em Geografia, Universidade Federal Fluminense. Campos dos Goytacazes, 2020.

DIAS, Jeniffer Silvana da Silva. A Política de Abastecimento de Gêneros Agrícolas e o Sistema Ceasa no Estado do Rio De Janeiro. Dissertação (Mestrado em Geografia) - Programa de Pós-Graduação em Geografia, Universidade Federal Fluminense. Campos dos Goytacazes, 2020.

BARRETO, Nina Maria de Souza. O Porto Do Açu (RJ): A Superexploração do Trabalho e os Conflitos Sócio-Espaciais. Dissertação (Mestrado em Geografia) - Programa de Pós-Graduação em Geografia, Universidade Federal Fluminense. Campos dos Goytacazes, 2020.

GONÇALVES, Mylena André. Centralidade Interurbana de Itaperuna - RJ: Uma Análise das Atividades de Comércio e Serviços. Dissertação (Mestrado em Geografia) - Programa de Pós-Graduação em Geografia, Universidade Federal Fluminense. Campos dos Goytacazes, 2020.

SOARES, Edmilson Campos. Cidade-Negócio e Responsabilidade Social Empresarial: Uma Análise Crítica da ONG Teto e Seus Financiadores. Dissertação (Mestrado em Geografia) - Programa de PósGraduação em Geografia, Universidade Federal Fluminense. Campos dos Goytacazes, 2020.

CUNHA, Andreia Ribeiro. "Do Rap ao Batidão” A Formação Político-Cultural Periférica e o Protagonismo da Mulher nos Movimentos Funk e Hip Hop: Contradições, Limites e Conflitos (Mestrado em Geografia) - Programa de Pós-Graduação em Geografia, Universidade Federal Fluminense. Campos dos Goytacazes, 2020.

CRUZ, Pamela de Souza da. Os Sentidos da Geografia Histórica na Revista Brasileira de Geografia (1939-1948) (Mestrado em Geografia) - Programa de Pós-Graduação em Geografia, Universidade Federal Fluminense. Campos dos Goytacazes, 2020.

WERNECK, Carlos Renato Ricardo. O Evento Catastrófico em Teresópolis/RJ e a Criação do Conjunto Habitacional Parque Ermitage, 2011-2019 (Mestrado em Geografia) - Programa de Pós-Graduação em Geografia, Universidade Federal Fluminense. Campos dos Goytacazes, 2020.

TAVARES, Thais Dias. "Respeite As Minas”: A Territorialidade Feminina no Movimento Hip-Hop em Campos dos Goytacazes e Macaé (Mestrado em Geografia) - Programa de Pós-Graduação em Geografia, Universidade Federal Fluminense. Campos dos Goytacazes, 2020.

PACHECO JUNIOR, Nelson Cortes. Da Capital às Tabancas: A Lugaridade entre os Guineenses e Missionários Protestantes em Guiné-Bissau (Mestrado em Geografia) - Programa de Pós-Graduação em Geografia, Universidade Federal Fluminense. Campos dos Goytacazes, 2020.

MARQUeS, Gabriel Guanabara Lemos. Plano Participativo de Mobilidade Urbana de Cabo Frio - RJ: Desafios e Possibilidades (Mestrado em Geografia) - Programa de Pós-Graduação em Geografia, Universidade Federal Fluminense. Campos dos Goytacazes, 2020.

SILVA, Anderson Luiz Barreto da. Religiões Afro-Brasileiras em Campos dos Goytacazes: Territórios, Conflitos e Resistência (Mestrado em Geografia) - Programa de Pós-Graduação em Geografia, Universidade Federal Fluminense. Campos dos Goytacazes, 2020.

ABREU, Juliana Silva de. Distribuição Geográfica da Pesca Marinha em Relação ao Recife Artificial Navio Victory 8b, Espírito Santo: Uma Análise a Partir do Conhecimento Tradicional de Pescadores Artesanais (Mestrado em Geografia) - Programa de Pós-Graduação em Geografia, Universidade Federal Fluminense. Campos dos Goytacazes, 2020.

OLIVEIRA, Pablo da Costa. Viabilidade da Pesca Artesanal Frente aos Rejeitos de Minério Lançados na Costa Norte do Espírito Santo: Uso do Conhecimento Tradicional (Mestrado em Geografia) - Programa de Pós-Graduação em Geografia, Universidade Federal Fluminense. Campos dos Goytacazes, 2020.

\section{9}

BASTOS, Raquel Figueira. Unidades de Conservação Ambiental no município de Campos dos Goytacazes - RJ (Mestrado em Geografia) - Programa de Pós-Graduação em Geografia, Universidade Federal Fluminense. Campos dos Goytacazes, 2019. 
BARBOSA, Antonio Ivo Gomes. Estimativa da Área Plantada de Cana-De-Açúcar em Campos dos Goytacazes - RJ Utilizando Sensoriamento Remoto (Mestrado em Geografia) - Programa de PósGraduação em Geografia, Universidade Federal Fluminense. Campos dos Goytacazes, 2019.

ARAUJO, Dayana Paes de. A Construção da Geografia Humana no Cruzamento do Evolucionismo Positivista com as Correntes Idealistas: Desarticulação do Projeto Científico Integrador? (Mestrado em Geografia) - Programa de Pós-Graduação em Geografia, Universidade Federal Fluminense. Campos dos Goytacazes, 2019.

COSTA, Layon Ignacio da Silva. Dinâmica Econômica e Locacional dos Investimentos na Indústria Fluminense (2010-2017) (Mestrado em Geografia) - Programa de Pós-Graduação em Geografia, Universidade Federal Fluminense. Campos dos Goytacazes, 2019.

CLARO JUNIOR, Jose Augusto. Estado e Dinâmica Econômica e Espacial da Indústria Automobilística no Brasil No Século XXI (Mestrado em Geografia) - Programa de Pós-Graduação em Geografia, Universidade Federal Fluminense. Campos dos Goytacazes, 2019.

SOUZA, Anderson Aparecido de. Políticas Territoriais e Segregação Socioespacial. Análise do Programa Minha Casa Minha Vida em Suzano-SP (Mestrado em Geografia) - Programa de Pós-Graduação em Geografia, Universidade Federal Fluminense. Campos dos Goytacazes, 2019.

VERLING, Yago de Souza. Zoneamento Ambiental do Trecho Fluminense da Bacia Hidrográfica do Rio Itabapoana - Brasil (Mestrado em Geografia) - Programa de Pós-Graduação em Geografia, Universidade Federal Fluminense. Campos dos Goytacazes, 2019.

SILVA, Ralfe de Souza Medeiros da. O Jogo De Xadrez como Recurso Metodológico na Elaboração de Mapas Mentais (Mestrado em Geografia) - Programa de Pós-Graduação em Geografia, Universidade Federal Fluminense. Campos dos Goytacazes, 2019.

NOGUEIRA, Fernanda de Faria Viana. De Quem São os Lugares na Cidade? Entendendo Trajetórias Gays em Campos dos Goytacazes - RJ (Mestrado em Geografia) - Programa de Pós-Graduação em Geografia, Universidade Federal Fluminense. Campos dos Goytacazes, 2019.

2018

ALMEIDA, Carolina Vieira Caldeira de Lima de Souza. Desenvolvimento de Práticas Educativas em Ensino de Geografia com alunos do $1^{\circ}$ ano do Ensino Médio da Rede Pública de Ururaí, Campos Dos Goytacazes (RJ), sob a Perspectiva do Fenômeno Inundação (Mestrado em Geografia) - Programa de Pós-Graduação em Geografia, Universidade Federal Fluminense. Campos dos Goytacazes, 2018.

TIRADENTES, Marlucia Degli Esposti. Vulnerabilidade e Risco de Inundação nos Municípios de Bom Jesus do Itabapoana (RJ) e Bom Jesus do Norte (ES) (Mestrado em Geografia) - Programa de PósGraduação em Geografia, Universidade Federal Fluminense. Campos dos Goytacazes, 2018.

QUEIROZ, Guilherme de Oliveira. A Política em Paul Vidal De La Blache: Resgate de um Ponto Chave (Mestrado em Geografia) - Programa de Pós-Graduação em Geografia, Universidade Federal Fluminense. Campos dos Goytacazes, 2018.

SILVA, Neusa Regina Barros Bastos da. Reflexões Sobre a Formação da Comunidade do Terminal Pesqueiro, em Farol de São Tomé, Campos dos Goytacazes - RJ e sua Identificação ao Lugar, Sob a Ótica Topofílica de Yi-Fu Tuan (Mestrado em Geografia) - Programa de Pós-Graduação em Geografia, Universidade Federal Fluminense. Campos dos Goytacazes, 2018.

SOUZA, Luma da Silva. Segregação Socioespacial e Política habitacional em Campos dos Goytacazes (RJ): Um estudo de caso sobre o Programa Morar Feliz em Ururaí (Mestrado em Geografia) - Programa de Pós-Graduação em Geografia, Universidade Federal Fluminense. Campos dos Goytacazes, 2018.

SILVA, Diogo Jordao. População em situação de rua e os usos do território: Uma análise das estratégias de sobrevivência na área central de Campos dos Goytacazes (Mestrado em Geografia) - Programa de Pós-Graduação em Geografia, Universidade Federal Fluminense. Campos dos Goytacazes, 2018.

MARTINS, Fabio Gustavo Pontes. A construção de condomínios no bairro Jockey Club: Um processo de exclusão socioespacial? (Mestrado em Geografia) - Programa de Pós-Graduação em Geografia, Universidade Federal Fluminense. Campos dos Goytacazes, 2018.

RANGEL, Arthur Nogueira. As ações dos grafiteiros e a produção da nova paisagem urbana de Campos dos Goytacazes (Mestrado em Geografia) - Programa de Pós-Graduação em Geografia, Universidade Federal Fluminense. Campos dos Goytacazes, 2018. 
TAVARES, Mariana Machado. A formação Geohistórica da Favela Baleeira em Campos dos Goytacazes - RJ (Mestrado em Geografia) - Programa de Pós-Graduação em Geografia, Universidade Federal Fluminense. Campos dos Goytacazes, 2018.

BARCELOS, Daniel Ribeiro. Guerra Fiscal e alienação do território no Estado do Rio de Janeiro (Mestrado em Geografia) - Programa de Pós-Graduação em Geografia, Universidade Federal Fluminense. Campos dos Goytacazes, 2018.

CUNHA, Thiago Ferreira da. Distribuição Espacial dos Casos de Notificações e Focos da Dengue no Município de Campos dos Goytacazes, Entre os Anos de 2011 a 2015, Utilizando Técnicas de Geoprocessamento (Mestrado em Geografia) - Programa de Pós-Graduação em Geografia, Universidade Federal Fluminense. Campos dos Goytacazes, 2018.

SANGUEDO, Juliana Bastos. Proposta de Construção de Índice Sintético de vulnerabilidade domiciliar a partir de dados dos censos demográficos brasileiros (Mestrado em Geografia) - Programa de PósGraduação em Geografia, Universidade Federal Fluminense. Campos dos Goytacazes, 2018.

BATISTA, Henrique Ferreira. Centro, Centralidade e Cidade Média: O Papel do Comércio e Serviços na Reestruturação da Cidade de Campos dos Goytacazes- RJ (Mestrado em Geografia) - Programa de Pós-Graduação em Geografia, Universidade Federal Fluminense. Campos dos Goytacazes, 2018.

SALES, Vanessa Barbosa. Indicador de Vulnerabilidade Socioambiental: Uma proposta metodológica em nível do indivíduo (Mestrado em Geografia) - Programa de Pós-Graduação em Geografia, Universidade Federal Fluminense. Campos dos Goytacazes, 2018.

SANTOS, Cleber de Jesus. Avaliação do Aquífero Raso no Parque São Benedito, Campos dos Goytacazes, Rj, Utilizando Geofísica e Exames Laboratoriais (Mestrado em Geografia) - Programa de Pós-Graduação em Geografia, Universidade Federal Fluminense. Campos dos Goytacazes, 2018.

LAMMLE, Luca. Impactos de Obras costeiras na linha de costa: O caso do Porto do Açu, São João da Barra, RJ (Mestrado em Geografia) - Programa de Pós-Graduação em Geografia, Universidade Federal Fluminense. Campos dos Goytacazes, 2018.

2017

COIMBRA, Thaina Santos. Geografia Com Tic's Cotidianas (Mestrado em Geografia) - Programa de Pós-Graduação em Geografia, Universidade Federal Fluminense. Campos dos Goytacazes, 2017.

AZEVEDO, Maria Catarina da Silva. As Transformações na Cobertura, Uso e Ocupação da Terra e Suas Consequências Sobre os Canais Fluviais: Análise das Inundações na Bacia do Rio Mataruna/ Araruama - RJ (Mestrado em Geografia) - Programa de Pós-Graduação em Geografia, Universidade Federal Fluminense. Campos dos Goytacazes, 2017.

CRESPO, Romulo De Almeida Beraldi. Instituindo a Aproximação Entre os Profissionais de Geografia e a Observação de Campo: A Discussão de Diferentes Tipologias de Trabalho de Campo Tendo Como Área de Estudo a Lagoa do Vigário em Campos dos Goytacazes-RJ (Mestrado em Geografia) - Programa de Pós-Graduação em Geografia, Universidade Federal Fluminense. Campos dos Goytacazes, 2017.

SILVA, Joilson Bessa da. Salas de Cinema em Campos dos Goytacazes: Lugar, Sociabilidade e Políticas Culturais - da década de 1960 aos anos 2010 (Mestrado em Geografia) - Programa de Pós-Graduação em Geografia, Universidade Federal Fluminense. Campos dos Goytacazes, 2017.

GAMA, Joao Penido. Técnica Survey Para Análise Espacial e Perfil dos Usuários: Uma Aplicação Para a Área Central de Niterói/RJ (Mestrado em Geografia) - Programa de Pós-Graduação em Geografia, Universidade Federal Fluminense. Campos dos Goytacazes, 2017.

FERES, Vinicius Soares Rangel Gomes. O Circuito inferior da economia urbana em Campos dos Goytacazes (Mestrado em Geografia) - Programa de Pós-Graduação em Geografia, Universidade Federal Fluminense. Campos dos Goytacazes, 2017.

CHAMBELA, Fernanda Aparecida. Análise da Rede Geográfica e da Perda de Densidade da Associação de Mulheres Rurais do Município de Espera Feliz - MG (Mestrado em Geografia) - Programa de PósGraduação em Geografia, Universidade Federal Fluminense. Campos dos Goytacazes, 2017.

GOVEIA, Luis Alberto Miranda. A internacionalização das empresas brasileiras durante os governos de Fernando Henrique Cardoso e Luiz Inácio Lula da Silva (Mestrado em Geografia) - Programa de Pós-Graduação em Geografia, Universidade Federal Fluminense. Campos dos Goytacazes, 2017.

ALVES, Priscila Viana. Experiências Poéticas em Porto Alegre: uma leitura geográfica de Mario Quintana (Mestrado em Geografia) - Programa de Pós-Graduação em Geografia, Universidade Federal Fluminense. Campos dos Goytacazes, 2017. 
PERES, Jose Felippe da Silva. Produção e Fragmentação do Espaço Urbano e o Direito à Cidade: Uma Analise do Programa "Morar Feliz" entre os Moradores dos Conjuntos Tapera II E Ururai II (Mestrado em Geografia) - Programa de Pós-Graduação em Geografia, Universidade Federal Fluminense. Campos dos Goytacazes, 2017.

COUTINHO, Edlane da Cruz da Silva. Geografia nas Provas do Enem: Abordagens Para Uma Compreensão Interdisciplinar (Mestrado em Geografia) - Programa de Pós-Graduação em Geografia, Universidade Federal Fluminense. Campos dos Goytacazes, 2017.

CRUZ, Maria Cecilia Soares. Território e Sindicalismo no Brasil: Uma Análise a Partir do Sindicato dos Petroleiros do Norte Fluminense (Mestrado em Geografia) - Programa de Pós-Graduação em Geografia, Universidade Federal Fluminense. Campos dos Goytacazes, 2017.

FIRMO JUNIOR, Reginaldo. Uso de Diferentes Representações Cartográficas no Ensino de Geografia no Ensino Médio: um estudo de caso no Colégio Estadual Dr. Phillippe Uebe, Campos dos Goytacazes-RJ (Mestrado em Geografia) - Programa de Pós-Graduação em Geografia, Universidade Federal Fluminense. Campos dos Goytacazes, 2017.

AMARAL, Cinthia Maria. Áreas de Risco e Vulnerabilidade Social em Áreas Urbanas: Soluções de Mapeamento Com Técnicas de Geotecnologias Para Viçosa-MG (Mestrado em Geografia) - Programa de Pós-Graduação em Geografia, Universidade Federal Fluminense. Campos dos Goytacazes, 2017.

VIRTUOSO, Anadelson Martins. Mapeamento da Cobertura e Uso da Terra Nas Faixas Marginais de Proteção do Rio Muriaé no Estado do Rio De Janeiro (Mestrado em Geografia) - Programa de PósGraduação em Geografia, Universidade Federal Fluminense. Campos dos Goytacazes, 2017.

VEIGA, Ronaldo de Souza. Uso e Ocupação da Terra no Município de Porto Seguro, BA: Uma Análise Geoambiental Multitemporal (1985-2016) (Mestrado em Geografia) - Programa de Pós-Graduação em Geografia, Universidade Federal Fluminense. Campos dos Goytacazes, 2017.

PAES, Raquel da Silva. Agricultura Familiar Versus Salinização: Uma Abordagem Etnopedológica no Norte Fluminense (Mestrado em Geografia) - Programa de Pós-Graduação em Geografia, Universidade Federal Fluminense. Campos dos Goytacazes, 2017.

SANT ANNA, Aline Guimaraes de Souza. As Praças e os Conteúdos das Desigualdades Socioespaciais Urbanas em Campos dos Goytacazes-RJ (Mestrado em Geografia) - Programa de Pós-Graduação em Geografia, Universidade Federal Fluminense. Campos dos Goytacazes, 2017.

RAIMUNDO, Amaral Morais. Geograficidades dos Pescadores Artesanais do Médio Inferior do Rio Paraíba do Sul (Mestrado em Geografia) - Programa de Pós-Graduação em Geografia, Universidade Federal Fluminense. Campos dos Goytacazes, 2017.

FARIA, Raphael Felix Ribeiro. Passos e Descompassos da Política de Mobilidade Urbana em Cidades de Porte Médio: O Sistema de Transporte Público BRT em Uberaba-MG (Mestrado em Geografia) - Programa de Pós-Graduação em Geografia, Universidade Federal Fluminense. Campos dos Goytacazes, 2017.

2016

CORREA, Sueleni Carvalho Fontes. Contribuição Aos Estudos Geomorfológicos e Sedimentológicos na Lagoa de Carapebus, RJ (Mestrado em Geografia) - Programa de Pós-Graduação em Geografia, Universidade Federal Fluminense. Campos dos Goytacazes, 2016.

LEANDRO, Assis Rangel. Impactos Socioeconômicos da Implantação do Complexo Logístico e Industrial do Porto do Açu no Distrito de Pipeiras São João da Barra - RJ (Mestrado em Geografia) - Programa de Pós-Graduação em Geografia, Universidade Federal Fluminense. Campos dos Goytacazes, 2016.

GAMA, Everton dos Santos da Silva. Zoneamento Ambiental do Município de Campos dos Goytacazes-RJ (Mestrado em Geografia) - Programa de Pós-Graduação em Geografia, Universidade Federal Fluminense. Campos dos Goytacazes, 2016.

TAVARES, Jessica Monteiro da Silva. Movimentos Pendulares de Estudantes na Região Norte Fluminense (Mestrado em Geografia) - Programa de Pós-Graduação em Geografia, Universidade Federal Fluminense. Campos dos Goytacazes, 2016.

RODRIGUES, Igor Paolo Ribeiro Dias. Território e Poder: As Elites e a Organização do Território em Campos dos Goytacazes (Mestrado em Geografia) - Programa de Pós-Graduação em Geografia, Universidade Federal Fluminense. Campos dos Goytacazes, 2016.

BARBOSA, Ivo Francisco. A Produção do Espaço Urbano em Aparecida - SP: Agentes e Processos (Mestrado em Geografia) - Programa de Pós-Graduação em Geografia, Universidade Federal Fluminense. Campos dos Goytacazes, 2016. 
CONCEICAO, Raphael Neves da. Leituras Geohistóricas da Paisagem da Baixada Campista (Mestrado em Geografia) - Programa de Pós-Graduação em Geografia, Universidade Federal Fluminense. Campos dos Goytacazes, 2016.pre

SOBRE O AUTOR

Marcelo Werner da Silva (SILVA, M. W.). Coordenador do Programa de Pós-Graduação em Geografia da Universidade Federal Fluminense, em Campos dos Goytacazes (UFF/Campos). Coordenador do Grupo de Estudos e Pesquisas de Geografia Histórica (Geohistórica). 